Palaeohispanica 2, (2002), pp. 333-353

\title{
INSCRIPCIÓN IBÉRICA PROCEDENTE DE LA JONCOSA (JORBA, BARCELONA)
}

\author{
$\mathrm{M}^{\mathrm{a}}$ Isabel Panosa \\ a Bertha Untermann, \\ in memoriam
}

\section{INTRODUCCIÓN}

Se presenta en este artículo una inscripción inédita grabada sobre una jarra de cerámica gris con dos asas. El carácter del documento es excepcional por contener un texto de diez líneas conservadas, lo que no es precisamente habitual en soportes cerámicos. El ejemplar más cercano de este tipo es el conocido rhyton de Ullastret (MLH III, C.2.8).

Hay que destacar en la pieza la presencia de nombres propios junto a elementos de la lengua común cuyo significado se nos escapa. También es importante señalar la presencia del vocablo iustir, que bajo esta forma o la de su variante iunstir suele aparecer en textos largos, especialmente en láminas de plomo y cerámicas pintadas. Esta forma también está presente en el ya citado rhyton de Ullastret junto a neitin, dando el compuesto antroponímico neitiniunstir.

Cabe decir que el epígrafe debe ser sometido a un proceso ulterior de limpieza y restauración, por lo que se espera poder completar en un futuro próximo las lagunas derivadas de una observación fragmentaria y limitada por el estado de conservación de la pieza en el momento del

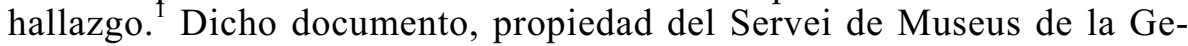
neralitat de Catalunya, será depositado próximamente en el Museo Comarcal de Anoia (Igualada).

\section{LUGAR Y CIRCUNSTANCIAS DEL HALLAZGO}

La inscripción fue hallada durante una excavación de urgencia llevada a cabo entre los meses de Junio y Septiembre de 1999 en la zona afectada por las obras de construcción de la autovía N-II (que une Barcelona con Lérida) a su paso por el municipio de Jorba (comarca de Anoia,

\footnotetext{
${ }^{1}$ Agradezco la atención y la ayuda prestada por el arqueólogo Josep Serra (de la empresa Atena 2000 SL de Igualada), quien puso a mi disposición el documento y todos los medios a su alcance para permitirme su estudio.
} 
provincia de Barcelona). Jorba se encuentra en la cuenca del río Ódena, a unos $7 \mathrm{~km}$. al noroeste de Igualada.

\section{EL YACIMIENTO}

Los trabajos de excavación fueron realizados por la empresa Atena $2000 \mathrm{SL}$ en la zona que iba a ser ocupada por un tramo de la mencionada autovía. A raíz de la intervención se sacó a la luz un asentamiento ibérico de tipo rural, con un área de hábitat y otra de almacenaje. Los materiales exhumados permiten atribuir al yacimiento un período de habitación que va desde el siglo IV hasta fines del II o principios del I a.C. El área de hábitat delimitada tenía aproximadamente $120 \mathrm{~m}^{2}$.

Durante la intervención se pudieron excavar 20 silos, 3 habitaciones, un vertedero y una serie de muros dispersos, cuya distribución puede apreciarse en la figura 1:

Hay que tener en cuenta que la extensión global del asentamiento debió de ser mayor, con numerosas fases de ocupación. Sin embargo, gran parte de la superficie había quedado prácticamente destruida en el momento de la excavación.

\section{CONTEXTO ARQUEOLOGICO Y CRONOLOGIA DE LA INSCRIPCION}

La inscripción apareció en la zona del vertedero, que contenía otros materiales cerámicos: fragmentos de ánforas Dressel $1 \mathrm{~A}$, cerámica de Cales, cerámica ibérica pintada, cerámica hecha a mano, cerámica común hecha a torno, fragmentos de morteros, piezas metálicas $\mathrm{y}$, en el estrato superficial, algunas monedas acuñadas en la Península y en varios puntos de la cuenca mediterránea. De las cecas locales o más o menos próximas al yacimiento tan sólo existe un ejemplar con leyenda $P \uparrow M E<\angle \Gamma$. Todas estas emisiones se fechan a finales del siglo II a.C.

El conjunto de materiales que acompañaban al documento epigráfico permite fecharlo dentro de la segunda mitad del siglo II a.C.

\section{EL SOPORTE}

Se trata de una jarra bitroncocónica con dos asas, de cerámica gris fabricada a torno. Se conserva de ella buena parte de la mitad superior, así como algunos fragmentos pertenecientes al pie y a la panza. Se comprueba que es una producción local, tal y como corrobora la pasta del recipiente. Además, se remarca el hecho de que se trata de un ejemplar único en su tipología y que parece haber sido fabricado expresamente para una ocasión muy concreta, posiblemente un obsequio con dedicatoria.

La pieza, que se puede apreciar en la figura 2, ha sido provisionalmente recompuesta a partir de varios fragmentos para permitir la restitución del texto. De esta recomposición se deduce un diámetro de la boca de $14,9 \mathrm{~cm}$. y una altura de la mitad superior de $10 \mathrm{~cm}$. aproximadamente. Se calcula una altura total de unos $20 \mathrm{~cm}$. 


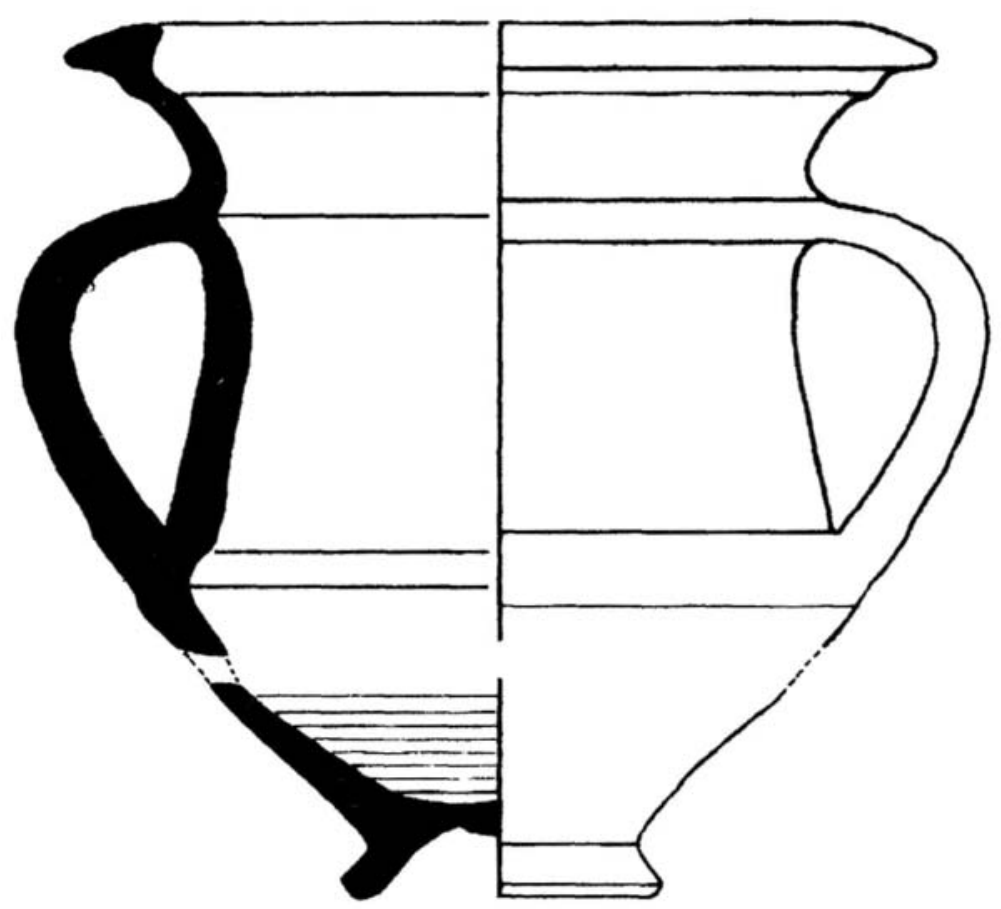

Fig. 2.- Dibujo de la jarra bitroncocónica con inscripción (Atena 2000 SL)

\section{EL TEXTO}

Afortunadamente, éste corre precisamente por la pared externa de la mitad superior del recipiente, que es la que se ha conservado en gran parte. No obstante, se ha perdido la zona adyacente a la segunda asa.

El texto fue marcado con anterioridad a la cocción de la cerámica. Tal como se observa en la figura 3 y en las fotografías, se desarrolla a lo largo de diez líneas que se distribuyen de la siguiente forma:

1) La primera línea comienza justo en el labio inferior de la jarra y por tanto muestra una posición inclinada de casi $90^{\circ}$ respecto a las otras líneas.

2) La segunda y tercera líneas están grabadas a continuación de la primera, en el cuello y separadas de las siguientes por una moldura horizontal en el recipiente.

3) Las siete líneas restantes se sitúan por debajo de la citada moldura, llegando justo hasta el surco que abre paso a la panza. Dos trazos verticales incisos a ambos lados del asa delimitan, respectivamente, el inicio y el final de cada una de estas siete líneas. 
Junto a los fragmentos ensamblados se cuenta otro fragmento aislado con signos, pero es imposible situarlo en su ubicación original dentro del texto seguido.

El tamaño de las letras oscila entre los 4 y los $7 \mathrm{~mm}$ de altura. Los trazos son rectos y claros, aunque no muy profundos, lo que dificulta la lectura de las letras más gastadas.

Se propone como transcripción la que sigue:

A: Texto largo

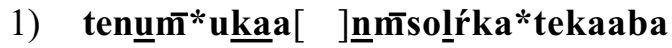

2) ]n̄itebanikate* uśiunbaŕr iekate eteolte eśarmīibabanmiiiekate[?

3) ]"libasetanebanite[?]tane eŕokarmibanitertanenite ketan[?

4) mśaseaŕmebetane[ ]snabetaneba[ ]aŕate[]ŕokarmieoŕosubeta

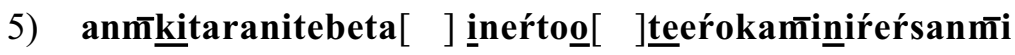

6) beśkiŕiustir*basetióa $\underline{\text { [ }}$ [ekate[ ]ten **akaribetainei

7) aŕŕseeŕatesuŕ *tebankite[ ]śírtebenm*

8) ]baŕsikeikanśarke*iskarkitar[ ]iaŕbo* śte

9) kan* ŕkualikiritekuosube[ ] $\underline{\text { śusen }}$ *uŕ *s

10) karakeatitibitirikater*rika[ ] $\underline{\text { banmini }[?] i e}$

B: Fragmento
1) $] \mathbf{r} *[$
2) ]nibabe[
3) ] aikae[

\section{A: TeXto largo}

Se ha perdido el inicio y el final de las líneas 2 y 3 del texto, además del inicio de la línea 8, en la que deben de faltar a lo sumo un par de signos. A continuación se comentan las secuencias identificadas en cada renglón y sus paralelos.

\section{Línea 1}

Se puede distinguir en primer lugar uka, cuya forma aparece también en un pondus de Sagunto (F.11.24).

En lírka se podría pensar otra alternativa para el primer signo: ba, con lo que resultaría baŕka, con paralelos en la inscripción rupestre de Masies de Roda (D.3.2), en una cerámica ática de Ullastret C.2.32) y en un plomo de Yátova (F.20.3,A-II,9), además del nombre propio baŕkakeŕ en una cerámica ática de Llinars del Vallès.

Al final tenemos tekaa (¿tal vez tekar, con la r6?) y el sufijo ba, que se puede comparar con tekar en un plomo de Ullastret $($ C.2.5,2). 
Inscripción ibérica procedente de La Joncosa (Jorba, Barcerlona)

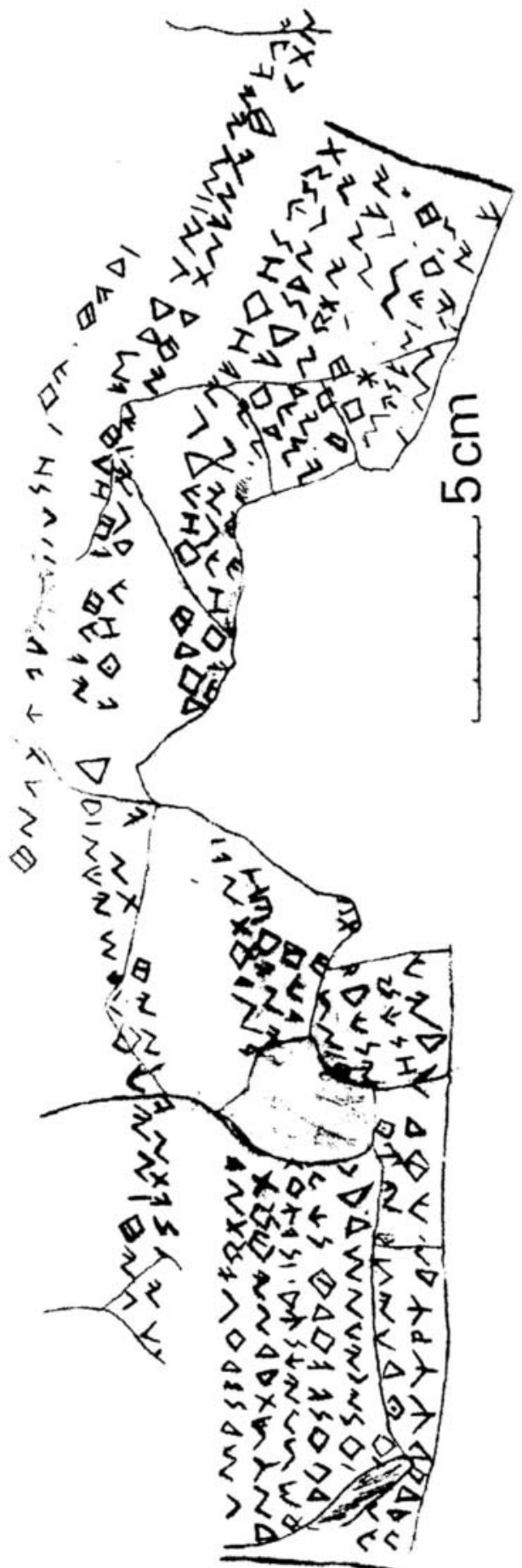




\section{Línea 2}

Se propone la siguiente segmentación: ]mi teban-i-kate* uśiunbaŕrie-kate eteol-te eś-ar-mi-ba-ban-mi-ie-kate.

El inicio de la línea se ha perdido. La parte conservada nos refleja en primer lugar el sufijo con valor posesivo $\mathbf{m i}$, que posiblemente acompañaba a una designación antroponímica. El último signo de la línea también podría ser i, dando kai.

A continuación se distinguen unas secuencias seguidas de kate. Obsérvese que en las líneas 6 y 10 aparece otra vez esta forma. Podría tratarse de un nombre común o bien de la secuencia de sufijos ka y te que suele aparecer detrás de nombres propios (MLH III, 1, p. 168).

El segmento teban, presente asimismo en la línea 7 de este mismo documento, aparece también en una estela de Sagunto (F.11.1) donde puede ser variante de eban, palabra típica del formulario sepulcral. Velaza (1994, p. 142 ss. y 1996.a, p. 262) atribuye a eban el valor de 'hijo', y a teban el de 'hija'. Esa posibilidad se podría confirmar si dispusiéramos del inicio de la línea, con la mención de dos nombres propios seguidos (la supuesta hija y su patronímico).

Respecto a uśiunbaŕr sólo existen paralelos para los siguientes segmentos: uś en un plomo de Orlell (F.9.1,A-6), iun en forma de ligadura en una tapa de cerámica de Badalona (Panosa 1993, n $\left.{ }^{\circ} 11.10\right)$, ${ }^{2}$ además de iunia en una cerámica ibérica de Cornellà (Panosa 2001, $\mathrm{n}^{\circ}$ $5.1){ }^{3}$ baŕ como texto único en un ánfora de Manresa (Panosa 1993, $\mathrm{n}^{\circ}$ 21.3) y como final de texto en una cerámica ática de Pontós (Panosa 2001, $\mathrm{n}^{\mathbf{0}}$ 7.1); véase también kubaŕ en el plomo de Badalona (supl. MLH III, C.8.3). Se sugiere la posibilidad de que se trate de un nombre propio.

En eteolte se distingue el elemento antroponímico ete, documentado por nombres como: etenbilos (Los Villares: F.17.1), eteiltuŕ (Yátova: F.20.3), eterintu (Sagunto: F.11.10), eteśike (Azaila: E.1.124) o Edeco (Turma Sall.) . A continuación vemos el segmento ol, que encontramos de forma aislada en una cerámica campaniense de Puiggraciós, (C.13.1), en una fusayola de Azaila (E.1.446) y en un peso de plomo de Arenys de Mar (C.6.1, aquí, al parecer, seguido de numerales). Por último vemos en la secuencia el conocido sufijo -te, bastante frecuente tras designaciones antroponímicas.

Al final de observa la secuencia eś-ar-mi-ba-ban-mi-ie. Se reconoce en ella dos veces el morfo posesivo $\mathbf{m i}$, combinado primero con ary luego con ban-, ambas cadenas conocidas en la mención de nombres de persona. Véase, por ejemplo, -banmi en la estela de Sinarcas (a continuación de la palabra seltar: F.14.1) y en una cerámica ibérica pintada de Sant Vicenç de Montalt (Panosa 1994, núm. 4). Cabe destacar también la secuencia de la fusayola de Palamós: tikirsbalauŕ.armi.banmi

\footnotetext{
${ }^{2}$ Untermann (supl. MLH III, C.8.8) lee imm.

${ }^{3}$ Véase asimismo iuntibilos en un plomo de Los Villares (F.17.1,A-9) y iuntegen en el plomo de El Cigarralejo (G.13.11). Ya se ha sugerido en otra ocasión la posibilidad de que iun sea variante del elemento antroponímico aiun.

${ }^{4}$ También tenemos oli ti en un ánfora de Pontós (Panosa 2001, p. 520, no 7.3).
} 
(C.4.2). En cuanto a eś, este segmento aparece de forma independiente en un plomo de Yátova (F.20.3, A-II,4) y en una cerámica campaniense de Pontós (Panosa 1993, $\mathrm{n}^{\mathrm{o}} 2.2$; como texto independiente o final de secuencia). El final coincide con banmiie en la línea 10.

\section{Línea 3}

Se sugiere como segmentación la que sigue: ]*libas-e-tane banite[?]tane eŕokaŕ-mi baniter-tanen ite-ketan[? (o ite-ke tan[?).

Se destaca en esta línea tane, repetido al menos tres veces y de forma alternada tras otras secuencias. Podría corresponder al elemento antroponímico tan o al elemento tanek. Por lo que respecta a ketan, hay que plantearse si se trata de un segmento independiente o bien se compone realmente del morfo o final ke (que en el habla correspondería posiblemente a una simple k) vinculado a ite, y luego, una vez más, tan. Citemos como paralelo próximo leśbaketante, del reciente plomo de la provincia de Granada (supl. MLH III, H.0.1, Aa-3), que podría admitir la siguiente segmentación: leśba-ke tan-te.

Por otro lado tenemos una serie de secuencias aparentemente relacionadas: ban-ite[?, ban-iter e ite, que se pueden comparar con ite (en anmkitaranite) en la línea 5. Una secuencia similar se ha documentado en el plomo de Empúries C.1.24,B/1-2: banitiŕ, donde la segmentación ban-itiŕ queda confirmada por la presencia del segundo segmento integrado en una secuencia más larga de la línea siguiente: itióroketebon. Este mismo plomo nos proporciona la secuencia iteŕibon (C.1.24,A-9), y el plomo de Camarasa presenta iteŕoke ${ }^{5}$ (supl. MLH III, D.13.1), lo que sugiere la existencia de dos variantes relacionadas: ite $(\mathbf{r} / \mathbf{r})$ e itiŕ. ${ }^{6}$ Por último, también tenemos banite en cerámicas ibéricas pintadas de Llíria (F.13.3,1, F.13.11 y F.13.33).

Para ]*libas puede pensarse en un final de nombre propio, por la presencia del elemento bas. El segmento anterior podría pertenecer a otro elemento antroponímico. Teniendo en cuenta que termina en -li, se ofrece la posibilidad de identificarlo con el único elemento atestiguado con seguridad hasta ahora con ese final: sili, lo que permitiría, por ejemplo, reconstruir la secuencia como (si)libas, seguido de un sufijo -e. Menos definitiva queda la dependencia o no de la secuencia siguiente tane respecto de (si)libas.

En último lugar nos queda por comentar la secuencia érokar-mìi, donde se distingue claramente el morfo posesivo - $\mathbf{m i}$. La misma secuencia se halla seguramente en la línea 4, (]ŕokarmi) y en la 5 (eŕoka-mi). Citemos como paralelo próximo éroke en el plomo de la Penya del Moro, en Sant Just Desvern (C.17.1,A-1). De forma análoga al paradigma de la raíz aŕ (o aŕe/aŕi, según la tabla de Untermann, MLH III,1, p. 181) o al de la raíz iŕ (de los que resultan formas como iŕe, aŕeka, aŕika,

\footnotetext{
${ }^{5}$ Seguramente comparable a las formas biteŕoke, bitíroke, biteŕokan y bitiŕokan de otros textos sobre plomo.

${ }^{6}$ Paralelas a las variantes biteŕ y bitír, a las que a menudo se suma la secuencia ok. Véase Untermann, MLH III, 1, pp. 184-185.
} 
iŕika o aŕikaŕ, entre otras), podríamos definir aquí la raíz eŕ seguida de la cadena okar ${ }^{7}$ y en último lugar el sufijo posesivo -mi.

\section{Línea 4}

La propuesta de segmentación es: móśas-e-aŕme-betan-e[ ]snabetan-eba[ ]aŕate-[ ]ŕokar-mi-e oŕosu-beta

Para mśas, seguida de un sufijo -e, no existen paralelos. Tan sólo contamos con inscripciones breves sobre cerámica campaniense procedentes de Azaila que contienen los dos primeros signos: móś (E.1.267 y E.1.268).

La secuencia aŕme tampoco está documentada, a no ser que sea variante de armi. Le sigue betan, probable elemento antroponímico que se repite dos veces más en esta línea. En la primera mención va seguido de una secuencia incompleta que comienza con -e (¿tal vez e(ba-), según se desprende de la segunda mención de este mismo elemento en la línea que se comenta?). El elemento betan está atestiguado por nombres como benebetan (F.13.12 y F.13.28, Llíria), sakaŕbetan (E.9.1, Benasal) y nmolbebetan (H.0.1, supl. MLH III).

La secuencia incompleta Jsna permanece sin interpretación. Y en cuanto a eba[ no se excluye la posibilidad de que se pueda completar como eba(n), lo que hipotéticamente sugeriría la noción de filiación, es más, tal vez una cadena de filiaciones con un elemento común: beta/betan que se prolongaría en esta línea.

La secuencia aŕate, también presente en la línea 7, admite la segmentación en aŕ y ate. Hallamos ate en un plomo de Yátova (F.20.3,BII,4), y atebarte en una cerámica campaniense de Solsona (D.5.1,2); aŕ es comparable a la raíz aŕe/aŕi definida por Untermann (MLH III,1, p. 181).

Seguidamente aparece la secuencia incompleta ]ŕrokar-mi-e. Se propone como inicio la letra e-, como en la secuencia prácticamente idéntica de la línea 3 (érokar-mi) y la forma análoga de la línea 5 (eŕoka-mi). El añadido del sufijo -e a la partícula mi está documentado en la secuencia no antroponímica aśune-mi-e de Ensérune (B.1.45; MLH III,1, p. 163; Velaza 1996.b, p. 47).

Al final de la línea, y seguido de beta, tenemos oŕosu, comparable tal vez a eŕeśu, en un plomo de Ullastret (C.2.3,A-5). Si admitimos la segmentación en oŕ-os-u, hallamos un paralelo directo en oŕ, presente en cerámica campaniense y cerámica gris de Azaila (E.1.162, .186 y .300). También cabe citar oŕotis, en una cerámica pintada de Llíria (F.13.3.8a). Por lo que se refiere al segundo segmento, os, tenemos: os en una cerámica campaniense y una fusayola de Azaila (E.1.185 y .447), osato en una cerámica gris de Ullastret (C.2.16) y ostaŕ en una cerámica campaniense de Solsona (D.5.1,1). No se excluye la posibilidad de interpretar oŕosu como un nombre propio.

\footnotetext{
${ }^{7}$ Con la posible segmentación -ok-aŕ. Otras formas documentadas que podrían pertenecer a la misma raíz son, por ejemplo: eŕeko, eŕeśu, eŕiar, érku o eŕto.
} 


\section{Línea 5}

La segmentación propuesta para esta línea, anmk̄kitar anite beta[ ]ịn eŕtoo[ ]te eŕoka-mi nírersan-mi, sugiere la mención de dos posibles nombres propios: anmkitar y niŕersan.

En primer lugar, anmkitar es seguramente un nombre de persona bimembre formado por anm $\overline{\overline{\mathbf{T}}}$ (quizás variante del elemento antroponímico an) y kitar (posible variante del elemento kitaŕ). Como paralelos están documentados los siguientes nombres: anmōberai (F.9.7,B-5), para el primer elemento, y kibaskitar (G.1.6, Alcoi), tu(r) skitar (F.9.3, Orlell), basto(k)kitaŕ (F.4.1, Coves de Vinromà) y Bastugitas (Turma Sall.), para el segundo. Obsérvese asimismo que kitar también aparece en la línea 8 , en el nombre propio iskarkitar.

Para anite (relacionado seguramente con la secuencia banite/baniter de la línea 5) se sugiere la partición en an-ite.

Una vez más hallamos la secuencia beta, presente también en la línea 4. Seguidamente, el texto se pierde hasta que aparece un final en -in, cuyo primer signo está incompleto, pero se puede reconocer con bastante seguridad como i.

Como posibles paralelos de eŕtoo[ véase eŕtoa[ en una lámina de bronce de Sagunto (F.11.29), eŕtois en una lámina de bronce de Betxí (F.7.2,3) y eŕtoska en un plomo de la provincia de Tarragona (C.0.2). Después de una laguna en la línea vemos el sufijo -te.

En cuanto a éroka-mi, véase el comentario expuesto en las líneas 3 y 4. Al final tenemos otro posible nombre propio, niŕersan, seguido de la partícula posesiva $-\mathbf{m i}$. Vemos niŕ dos veces como segmento final de secuencias más largas en un plomo de Yátova (F.20.1,A-I,1 y 2). ${ }^{8}$ Por otro lado tenemos niŕr[-]ŕekon en la inscripción rupestre de Cogul (D.8.1,c) y eśuŕeśuniŕ junto a ilereutiniŕ en un plomo de Pech Maho (supl. MLH II, B.7.34); san podría ser variante del elemento antroponímico sani.

\section{Línea 6}

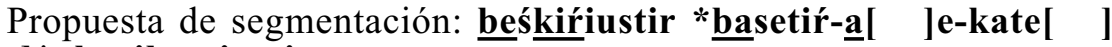
$\underline{\text { ten }[~] * a k a r i b e t a i n-e i}$

Existe lā posibilidad de considerar beśkiŕiustir como una designación antroponímica comparable al ya conocido neitiniunstir, interpretado como nombre propio. Así pues, según el contexto, la palabra iunstir puede funcionar unas veces como elemento antroponímico y otras como forma apelativa de la lengua. ${ }^{9}$ Para el caso de neitiniunstir la interpretación como nombre propio está clara porque se ha documentado el elemento neitin en otros compuestos antroponímicos. Sin embargo, para el caso que nos ocupa no disponemos de otros testimonios que aseguren que beśkir sea un elemento antroponímico. Queda abierta, pues, su interpretación.

\footnotetext{
${ }^{8}$ No se excluye, sin embargo, que en los dos ejemplos mencionados la $\mathbf{n}$ pertenezca a la secuencia precedente y que el segmento final sea en realidad -iŕ.

${ }^{9}$ Véase Untermann 2001, pp. 618-621, y las referencias bibliográficas citadas por el autor.
} 


\section{$M^{a}$ Isabel Panosa}

En la secuencia transcrita como *basetiŕ-a [ no está del todo claro el principio; es posible que en lugar de ba se halle aquí el signo o, algo mutilado, en cuyo caso la lectura sería osetiŕ-a[. En cuanto al último signo (a), si su identificación es correcta, podría tratarse de un sufijo como el que encontramos en nombres como ildun-iŕ-a (G.1.1,B-4/5, Alcoi), kuleśuŕi-a (D.7.1, Sidamon) y alostibaś-a-mi (B.1.274, Ensérune). ${ }^{10}$ Nótese también el segmento -iŕ́- en la secuencia que aquí comentamos, al igual que en el ya citado ildun-iŕ-a. Si se acepta la primera lectura, tenemos eŕbatebanbase como inscripción rupestre en $\mathrm{Er}$ (Campmajo y Untermann 1991, p. 46, no 2), arkiteibase en la inscripción sobre piedra de Cruzy (supl. MLH II, B.11.1), baśerke en cerámica pintada de Llíria $(\mathrm{F} .13,70)$, baseŕoke y baseŕokar en un plomo de Alcoi (G.1.1,A-3,4/5) y el probable nombre propio baseŕnmmlbe* en un plomo de Orlell (F.9.7, B-1). Para la lectura osetír-a no existen testimonios. Otra posibilidad para el último signo de esta secuencia es que sea $\mathbf{s}$, ofreciendo la siguiente transcripción basetiŕs. En ese caso, citemos tiŕs en un plomo de Ullastret (C.2.3,A-3).

Respecto a kate, véase el comentario de la línea 2. Después de una laguna se presenta la secuencia ten, seguramente incompleta; véase tena en un plomo de Yátova (F.20.3,B-II,10).

Por último, en *akaribetain-ei se diferencia, por un lado, akari y, por otro betain seguido del sufijo -ei, posible variante del final en -e. Compárese esta secuencia con akariśalir delante de numerales en otro plomo de Yátova (F.20.2,B-11,12), lo que hace pensar - al menos en este caso- en una forma apelativa por su conexión con śalir. A continuación, betain no dispone de paralelos exactos; tan sólo aparece la secuencia beta en una cerámica campaniense de Azaila (E.1.99) y en una estela de Binéfar (D.12.2). Es muy probable que esté relacionada con el elemento antroponímico betan (o betaŕ) que también aparece varias veces en la línea 4 y una vez en la 5 , aquí seguido de una terminación -in (habitualmente atribuida a nombres femeninos) y del sufijo -ei.

\section{Línea 7}

Propuesta de segmentación: aŕŕseeŕatesứr* teban kite[ ]śí́ teben $\mathbf{m}^{*}$

Destaquemos en primer lugar la presencia de teban detrás de una secuencia que podría interpretarse como designación antroponímica. Con todo, y aunque no deje de ser muy aventurada, se presenta la hipótesis de identificar a un nombre femenino junto con su patronímico seguido de la palabra 'hija' (teban). El primero sería la secuencia final de la línea anterior, akaribetain (o por lo menos betain), y el segundo, o patronímico, sería aŕŕseeŕatesuŕ. En esta última secuencia, cuya propuesta de segmentación es aŕŕse-eŕate-suŕr(i?), se identifican posiblemente los elementos antroponímico aŕs (aquí con ŕ duplicada, por razones que se desconocen, si no es que se trata de un error de escritura) y suŕ (variante de sor o soŕ). Véase ibeisur en Pech Maho (supl. MLH II, B.7.35 y .36), kuleśuŕi en Sidamon (D.7.1) y lauŕsu(r) en Pontós (C.3.1). Menos clara

\footnotetext{
${ }^{10}$ Véase el comentario de Untermann a este sufijo (que sustituiría a ar) en MLH III,1, p. 157.
} 
queda en este contexto la secuencia eŕate, que se podría segmentar en eŕ y ate. Esta misma forma se presenta también en la línea 4.

Por lo que respecta a kite[, véase: kidei en un plomo de Alcoi (G.1.1,A-I,7, después de la mención de śalir); kite repetida tres veces en secuencias más largas de una cerámica pintada de Llíria (F.13.4) y al inicio de un texto incompleto en cerámica común de Azaila; bankite y enkite en cerámicas pintadas de Alloza (respectivamente, E.4.2 y .3), y kiteibors en un plomo de Yátova (F.20.1,A-II,8).

A continuación se presenta un final -śír seguido de teben (¿variante de teban?), cuyo segundo signo está bastante borroso, y en último lugar $\mathbf{m}^{*}$ (tal vez $\left.\underline{\mathbf{m}}(\mathbf{i})\right)$. El primer segmento podría ser una variante del elemento antroponímico sir, atestiguado por los nombres: koŕasió en un plomo de la provincia de Tarragona (C.0.1) y kuleśir en plomos de Pech Maho (supl. MLH II, B.7.35 y .36).

\section{Línea 8}

Esta línea, cuya propuesta de segmentación es ]baŕsike-i kanśarke* iskarkitar[ ]iaŕbo* ś-te, permite identificar una serie de nombres propios.

El primero de ellos, con inicio mutilado, se compone de ]baŕ y sike, con terminación en -i. Se ofrecen dos posibles restituciones para el primer elemento: (a)baŕ o $(\mathbf{m} \overline{\mathbf{m}}) \mathbf{b a r}$. Para ambos, los paralelos en compuestos antroponímicos son numerosos, sobre todo con dicho elemento en primera posición: abaŕeśker y abaŕtaŕ en Llíria (respectivamente, F.13.1 y .2); abaŕtikeŕ en Pech Maho (supl. MLH II, B.7.37); mbaratin en Ensérune (B.1.283); Umarbeles (Turma Sall.), Umarillun (Turma Sall.) y Umargibas (Turma Sall.). El segundo elemento, sike, también está bastante documentado en compuestos antroponímicos, por ejemplo: sikeboneś en un plomo de Alcoi (G.1.6), sikeunin en una lápida de Sagunto (F.11.6), sikounin en un plomo de Empúries (C.1.6,B-1), eteśike en una cerámica campaniense de Azaila (E.1.124), sikaai en una moneda de Obulco (A.100) y Sicae en Portoalegre (II 169).

El segundo nombre, kanśarke*, está constituido por los elementos kanś (posible variante de kan o kani) y arke*. En cuanto a kans, contamos con la misma forma en una cerámica común de El Masnou (Panosa 1993, p. 180, no 3.2) y una cerámica campaniense de Azaila (E.1.262). Además tenemos: kaniberon en en plomo de La Bastida de les Alcuses (G.7.2,B-5), ganikbos en el plomo de El Cigarralejo (G.13.1) y kanisoŕ en un plomo de Pech Maho (supl. MLH II, B.7.35). El segundo elemento tal vez admita ser restituido como arke(r), aunque también es posible que el nombre termine con -e y que el signo perdido pertenezca a la secuencia siguiente. Compárese con los nombres siguientes, donde este elemento aparece en segundo lugar: abaŕkis en el plomo de Palamós (C.4.1), balkarki en la inscripción rupestre de Cogul (D.8.1), iltíraŕker en una moneda de Empúries (A.6) y bilo(s)aŕker en una cerámica gris de Cerdanyola (Panosa 1993, pp. 206-207, $\mathrm{n}^{\circ}$ 19.2).

El tercer nombre, iskarkitar, compuesto de iskar y kitar, presenta en primer lugar una posible variante del elemento antroponímico isker. En segundo lugar nos muestra el mismo elemento que encontrábamos en 
la línea 5 de este documento: anmkitar. Véase, pues, el comentario expuesto en ese lugar.

El cuarto y último podría ser restituido como iaŕbo(ŕ)-śte y definido como un compuesto de iaŕ (posible variante del elemento antroponímico iar), bo( ste, como en bor-ste en un plomo de Ullastret (C.2.3,A-2), bitar-ste y saltir-ste, ambas en un plomo de Yátova (F.20.2,A-2). El elemento iaŕr/iar está documentado en los nombres propios iaribeŕ en la estela de Caspe (E.13.1), ianbin y beŕian en estelas de Sagunto (respectivamente, F.11.4 y .10), iskeriaŕ en el fragmento de plomo de Llano de la Consolación (G.15.1) y en Corsyaninai en una inscripción de Sagunto con alfabeto latino (II 3903). El segundo elemento, boŕ, cuenta con testimonios muy numerosos. Baste mencionar, por ejemplo, aŕkebor (C.19.2, Els Monjos), beleśboŕ (C.4.1, Palamós) o biuŕboŕ (B.1.3, Ensérune), entre otros.

\section{Línea 9}

Se propone la siguiente segmentación: kan*ŕku alikirite-ku

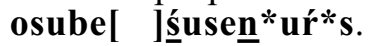

En la primera secuencia, el segundo signo está incompleto, pero es bastante seguro que corresponda a una n. Queda, en cambio, omitido el tercer signo. Propongo reconstruirlo como una $\mathbf{i}$, de lo que resultaría kan(i)ŕku. En ese caso se podría pensar en el segmento kan ${ }^{11}$ seguido de iŕku, donde se distinguiría a su vez la raíz iŕ seguida de un sufijo -ku. Véase kan como forma aislada en una cerámica campaniense y un pondus de Azaila (respectivamente, E.1.141 y .385), y tal vez en una cerámica campaniense de Empúries (C.1.6).

La secuencia siguiente, alikirite-ku, parece mantener concordancia con la primera por su final en -ku. No está claro si te es un morfo añadido o bien pertenece a la cadena que le precede, lo que recordaría a la conocida expresión toponímica usekerte-ku, con valor aproximado de 'en Osicerda'. Por lo demás, esta cadena permanece bastante oscura. Tan sólo existen algunos paralelos para segmentos cortos de ella: al como texto aislado en una cerámica campaniense y un pondus de Azaila (respectivamente, E.1.48 y .356), así como en una cerámica campaniense de Sant Boi de Llobregat (Panosa 1993, pp. 201-202, n $\left.{ }^{\circ} 15.2\right)$ y otra de Sant Feliu de Guíxols (Panosa 2001, p. 523-524, n ${ }^{\circ} 10.1$ ), y ]kiŕi[ en una cerámica campaniense de Ensérune (B.1.239).

Para osube[ existen paralelos si pensamos en una segmentación en os y ube. Para el primero encontramos os en una cerámica campaniense y una fusayola de Azaila (E.1.185 y .447), así como en una cerámica campaniense de Ensérune (B.1.99), osati en una cerámica gris de Ensérune (B.1.250), osato en una cerámica gris de Ullastret (C.2.16), osi[ en un plomo de Yátova (F.20.3,A-II,4) y ostaŕ en una cerámica campanien-

\footnotetext{
${ }^{11}$ Otra posibilidad, aunque menos probable, sería que el primer segmento representara al elemento antroponímico kani; sin embargo, es raro hallar el sufijo -ku detrás de nombres propios, y quizás deberíamos ver en esta secuencia completa más bien una expresión de la lengua común.
} 
se de Solsona (D.5.1,1). Para el segundo contamos con ube en una cerámica común de Azaila (E.1. 291), aunque de lectura algo dudosa. Si, en cambio, pensamos en una segmentación en osu y be[, podemos citar la secuencia oŕosu de la línea 4 de este documento.

Queda, por último, poco definida la secuencia lśsusen*uŕ*s, en la que se identifica posiblemente la raíz uŕ que encontramos en formas como uŕi o uŕke; śu también aparece como inicio de cadenas más largas: śu* [, śuate y śunuke en cerámica campaniense y común de Ensérune (respectivamente, B.1.133, .67 y .310), śumitatiniŕe en cerámica pintada de Llíria (F.13.5), śuśu[ en un plomo de Los Villares (F.17.2,A3) y śuśun en un plomo de Yátova (F.20.3,B-II,8).

\section{Línea 10}

La segmentación de esta línea (karakeatitibitirikater*rika[ ] banmiie) es más compleja, por lo que se prefiere comentar directamente las secuencias que se puedan individualizar.

Untermann (MLH III,1, pp. 184-185) definió un lexema con la forma biteŕ o bitiŕ que tal vez corresponde al que encontramos en esta línea con la forma bitir ${ }^{13}$ en bitirikater. Una parte de esta secuencia es comparable a la siguiente, *rika, que se podría reconstruir como (i)rika o (a)rika. Para kar contamos con ejemplos como texto único o inicio de secuencias más largas: kar en un pondus de Azaila (E.1.386), karkaśar y tal vez karkustiko en cerámica ática y pintada de Llíria (respectivamente, F.13.71 y .14), y karkankati en una cerámica campaniense de Badalona (Panosa 1993, pp. 192-193, $\mathrm{n}^{\circ}$ 11.7). Al final se reconoce, aunque con lectura dudosa, la cadena ban-mi seguida de -ie (o -i-e), si la transcripción es correcta. Compárese con ]ban-mi-ba-e en una cerámica pintada de Llíria (F.13.3,3,16) y con ba-ban-mi-ie casi al final de la línea 2.

\section{B: FRAGMENTO}

Las líneas de este fragmento estaban integradas en el texto largo que acabamos de comentar, pero no hay elementos para averiguar a qué altura de ese texto pertenecen.

Entre los signos conservados se puede comparar la secuencia ]nibabe de la segunda línea con babe en un pondus de Azaila (E.1.361); ]ai kae[ puede cotejarse con aikas y aikase en el plomo de Castellón (respectivamente, F.6.1,4 y 3), y aike en una cerámica campaniense de Azaila (E.1.25). Debido al estado fragmentario del texto, permanece oscura una posible relación de ]aikae[ o -ikae[ con el elemento antroponímico ike.

\footnotetext{
${ }^{12}$ Más hipotética sería la opción de relacionar śuse o śusen con el elemento antroponímico suise, puesto que faltan otros testimonios que lo corroboren y, además, se ignora si śu es aquí inicio de esta secuencia o final de otra que ha quedado mutilada.

${ }^{13}$ Con esta misma $\mathbf{r}$ se puede mencionar otro ejemplo: bitirebabosin en el plomo de Los Villares citado supra (F.17.2,A-4).
} 


\section{CONCLUSIÓN}

No cabe duda de que estamos ante una pieza de gran valor que, por su tipología cerámica y la longitud del texto, representa un unicum en el área ibérica en general. Como es sabido, a parte de los plomos y del rhyton de Ullastret, tan sólo se suelen encontrar textos largos en la cerámica ibérica pintada.

Según se ha dicho anteriormente, se destaca el hecho de que la pieza fue fabricada en el área geográfica inmediata; al menos así lo confirma la pasta empleada para su elaboración. Y puesto que los signos han sido marcados previamente a la cocción, se deduce que la inscripción fue realizada por algún miembro de la comunidad local y probablemente en el mismo taller de fabricación del recipiente. Lo que obviamente no se puede asegurar es si el autor pertenecía a este mismo asentamiento o a algún otro de sus proximidades.

En el texto se mencionan, junto a expresiones de la lengua común, una serie de nombres propios que en determinados casos podrían ser femeninos (como por ejemplo betain o akaribetain en la línea 6). Algunos de estos nombres podrían estar relacionados entre sí, posiblemente por vínculo de parentesco, si admitimos la interpretación sugerida para los términos eba(n) (en la línea 4) y teban (en las líneas 2 y 7), así como las coincidencias del elemento beta/betan en nombres encadenados que se observan en la línea 4. También kitar aparece en dos compuestos antroponímicos de este documento (anmkitar e iskarkitar).

En cuanto a la finalidad del objeto y de su inscripción se ofrece ante todo la posibilidad de un regalo con una larga dedicatoria. Caso de ser así, quedaría por averiguar quién es el redactor del texto y quién es destinatario o la destinataria de este obsequio, en el caso de ir destinado a una sola persona. ${ }^{14}$

En el rhyton de Ullastret aparece la palabra ekiaŕ — relacionada con la noción de 'hacer' - junto con varios posibles nombres propios: neitiniunstir, ilbikon, akerbikir, auskeikar. Entre ellos, uno debe corresponder sin duda al autor de la pieza y al menos otro a su destinatario. Así pues, también en este ejemplo hay que interpretar el texto como una dedicatoria.

Otro ejemplo, aunque con una inscripción de mayor brevedad, es el fragmento de un recipiente globular de cerámica gris ibérica procedente de Turó de ca n'Olivé, en Cerdanyola (Panosa 1993, pp. 206-207, ${ }^{\circ}$ 19.2), donde se lee bilo(s)arkerte(e)kiar. En este texto, al parecer completo, se observa la mención de una persona, bilo(s)arker seguida del sufijo -te y de la misma expresión (e)kiar, aludiendo seguramente al autor de la pieza.

En el texto de Jorba, la forma ekiaŕ está ausente, aunque podría estar contenida en la parte perdida del documento. Por último, se puede destacar que la datación de esta cerámica en el siglo II a.C. coincide con

\footnotetext{
${ }^{14}$ No se excluye la posibilidad de haber sido destinado a más personas (una familia, por ejemplo).
} 
la que Guadan y Villaronga (1964) propusieron también para el rhyton de Ullastret. ${ }^{15}$

\section{BIBLIOGRAFIA}

CAMPMAJO, P. - UntermanN, J. (1991): "Corpus des gravures ibériques de Cerdagne", Ceretania 1, pp. 39-59.

GUADAN, A.M. - VILlaRONGA, L. (1964): "En torno a la interpretación del grafito en un rhyton de Ullastret", Archivo Español de Arqueología 37, pp. 33-39.

MLH II = UNTERMANN, J. (1980)

MLH III = UNTERMANN, J. (1990)

PANOSA, Ma I. (1993): "Nuevas inscripciones ibéricas de Cataluña", Complutum 4, pp. 175-222.

(1994): "Els càlats i l'escriptura ibèrica", Lauro 8, pp. 3-7.

(2001): "Novedades de epigrafía ibérica en Cataluña y algunos aspectos metodológicos", en: F. Villar y $\mathrm{M}^{\mathrm{a}} \mathrm{P}$. Fernández (eds.), Religión, lengua y cultura prerromanas de Hispania. Actas del VIII Coloquio Internacional sobre Lenguas y Culturas Prerromanas de la Península Ibérica, Salamanca 2001, pp. 511-540.

PericAY, P.- MALUQuer DE MOTES, J. (1963): "Problemas de la prehistoria y de la arqueología catalanas", $2^{\circ}$ Simposium de Prehistoria Peninsular, Barcelona, pp. 101-143.

UntermanN, J. (1980): Die Inschriften in iberischer Schrift aus Südfrankreich, Dr. Ludwig Reichert Verlag, Wiesbaden.

(1990): Die iberische Inschriften aus Spanien, Dr. Ludwig Reichert Verlag, Wiesbaden.

(2001): "Algunas novedades sobre la lengua de los plomos ibéricos", en: F. Villar y M ${ }^{\mathrm{a}} \mathrm{P}$. Fernández (eds.), Religión, lengua y cultura prerromanas de Hispania. Actas del VIII Coloquio Internacional sobre Lenguas y Culturas Prerromanas de la Península Ibérica, Salamanca 2001, pp. 613-627.

VELAZA, J. (1994): "Iberisch eban, teban", ZPE 104, pp. 142-150.

(1996.a): Epigrafía y lengua ibéricas, Arco/Libros SL, Cuadernos de Historia, Madrid.

(1996.b): "Epigrafía funeraria ibérica", ELEA 2, pp. 251-282.

$M^{a}$ Isabel Panosa

Universitat Internacional de Catalunya

e-mail:mpanosa@cir.unica.edu

\footnotetext{
${ }^{15}$ Pericay y Maluquer (1963, p. 107) prefieren una cronología más antigua: la transición del siglo IV al III a.C.
} 


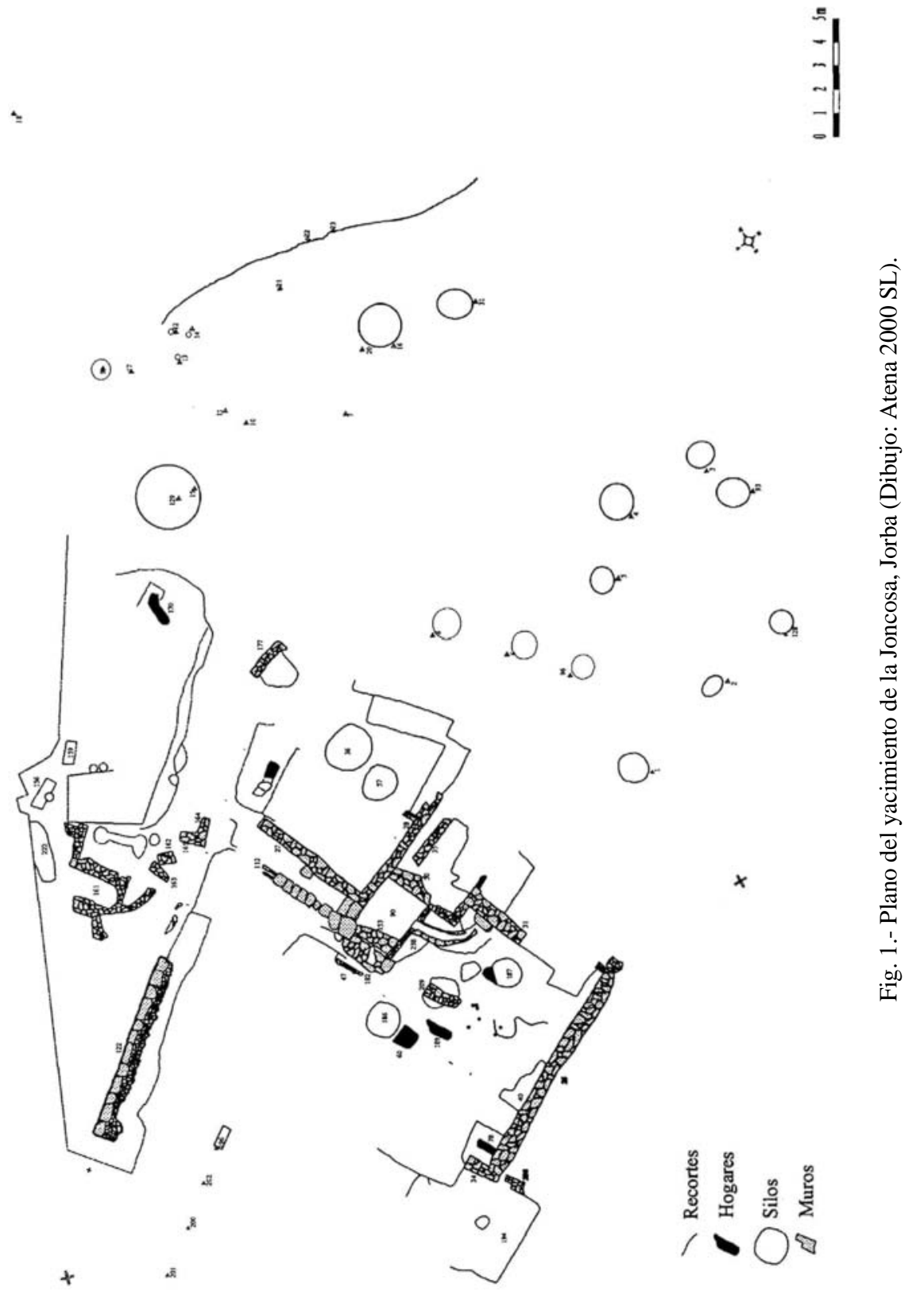


Inscripción ibérica procedente de La Joncosa (Jorba, Barcerlona)

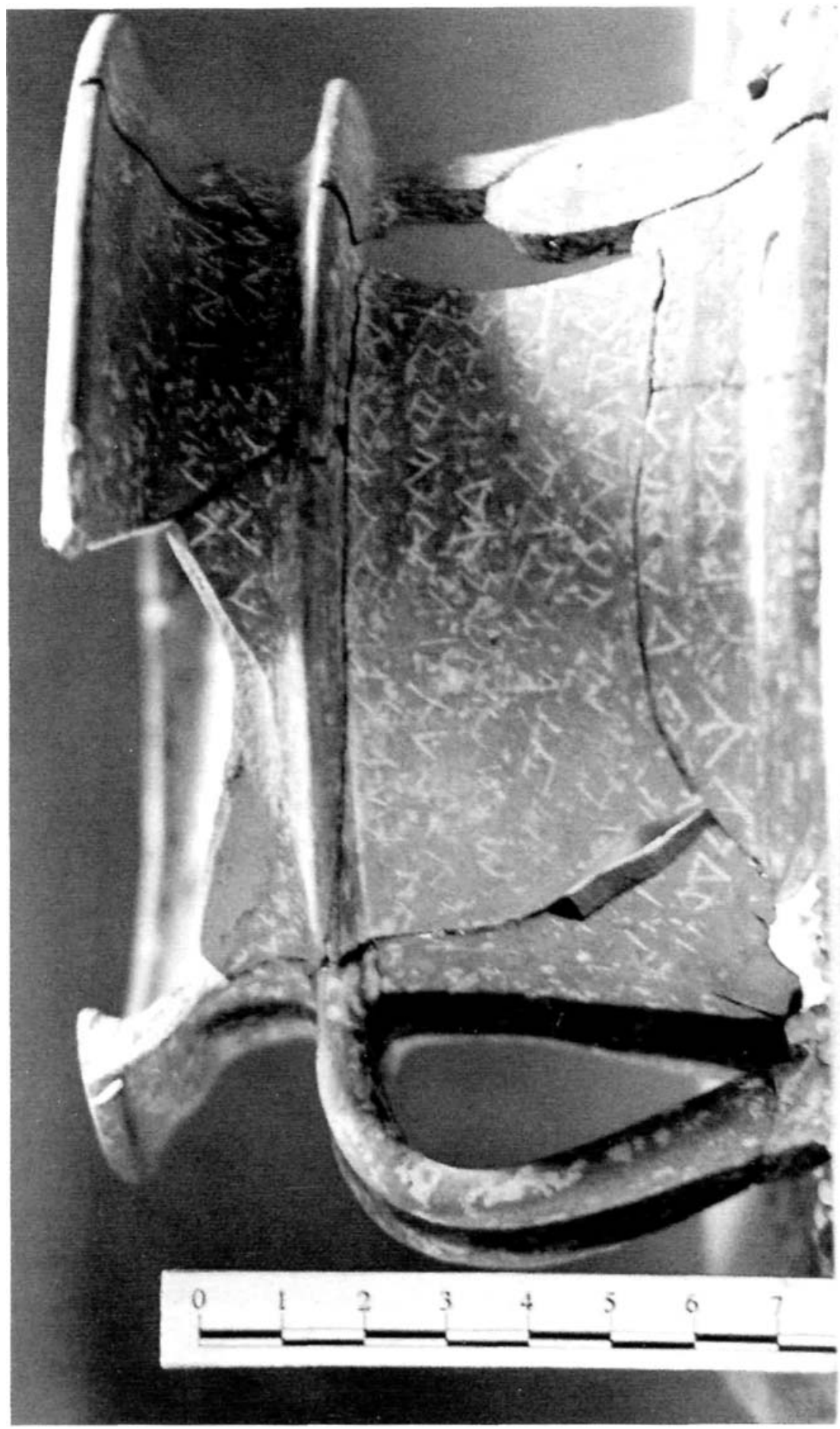

무류. 


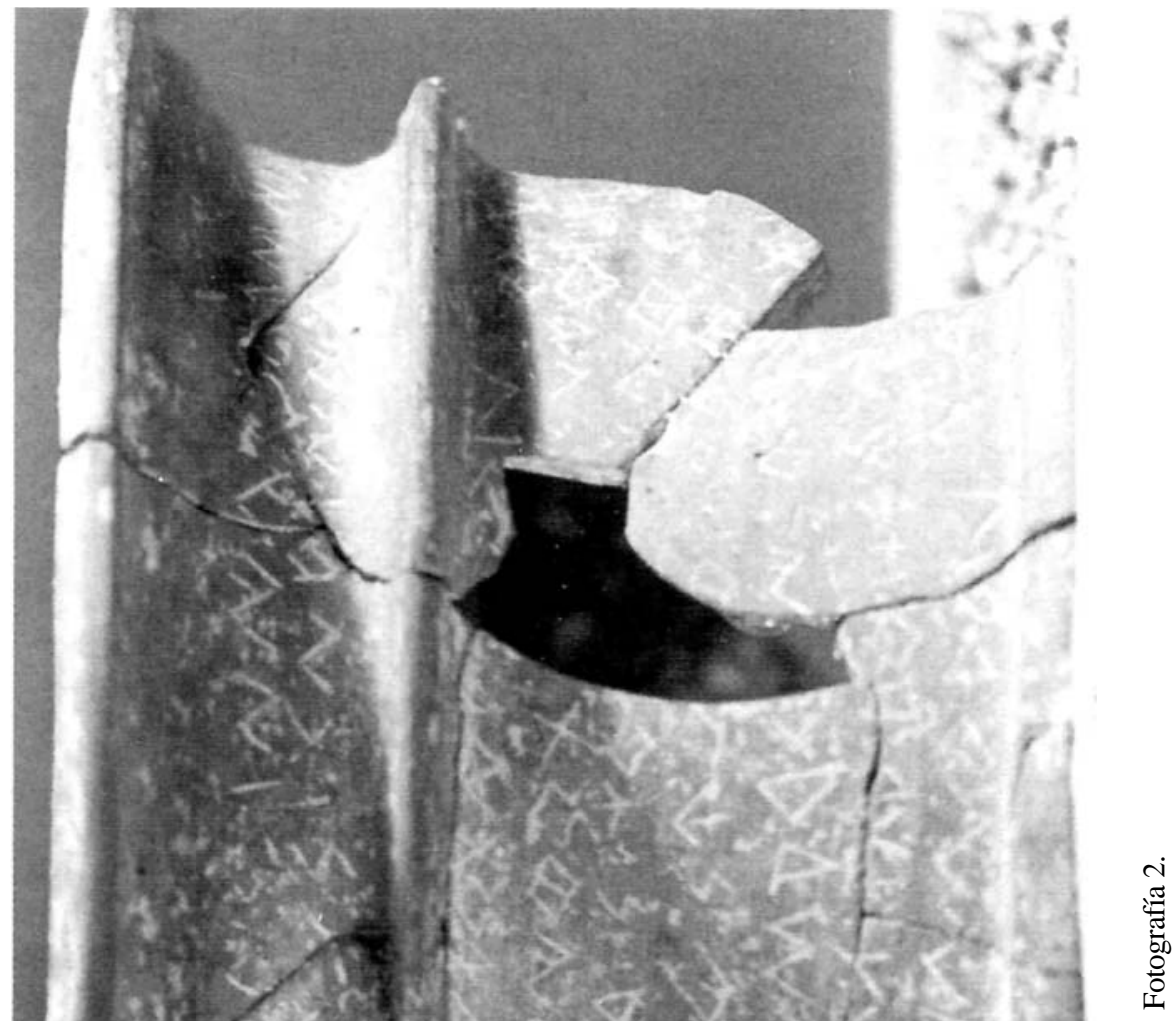

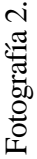


Inscripción ibérica procedente de La Joncosa (Jorba, Barcerlona)

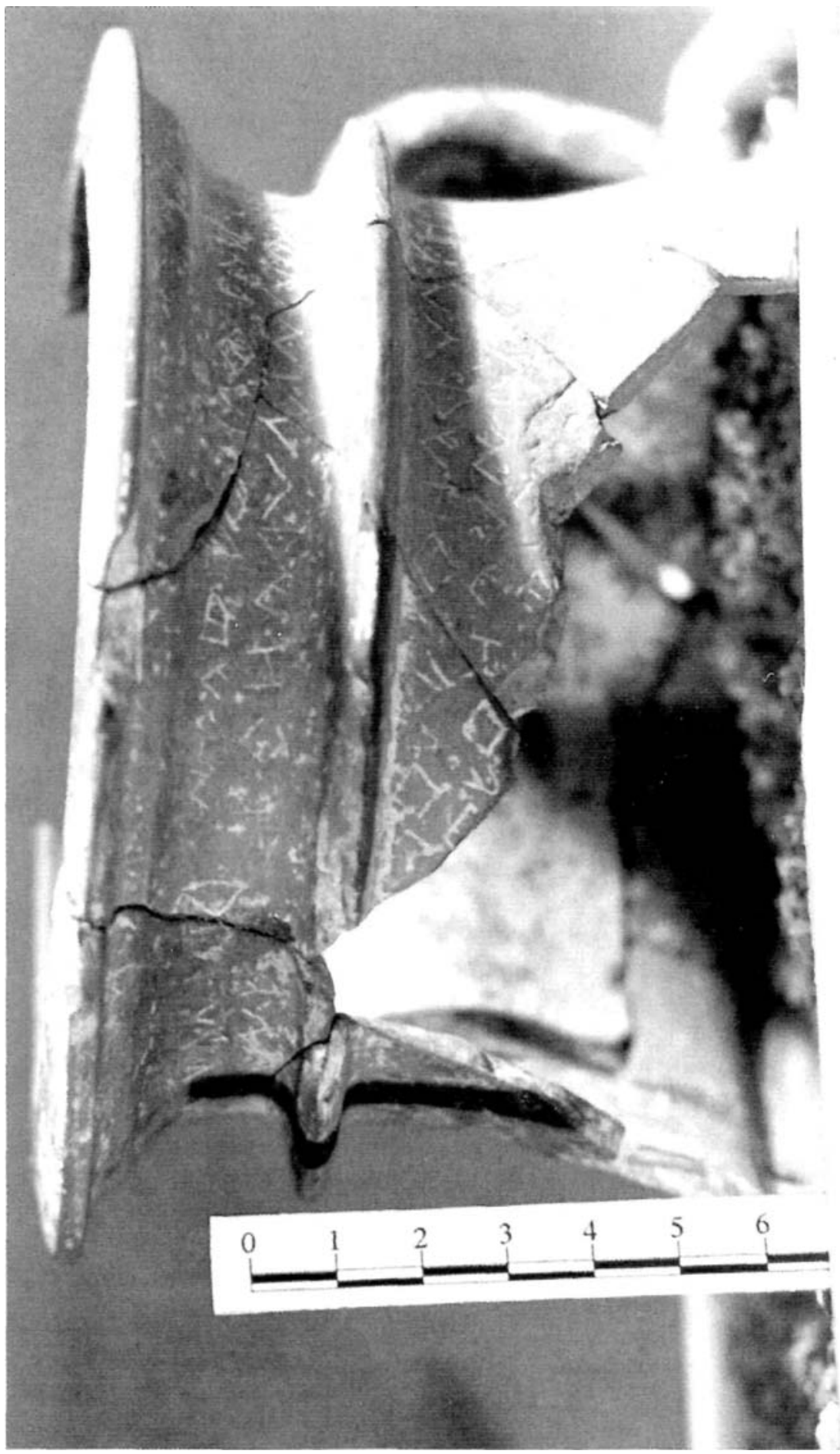

0
0
0
0
0
0
0
0
0 


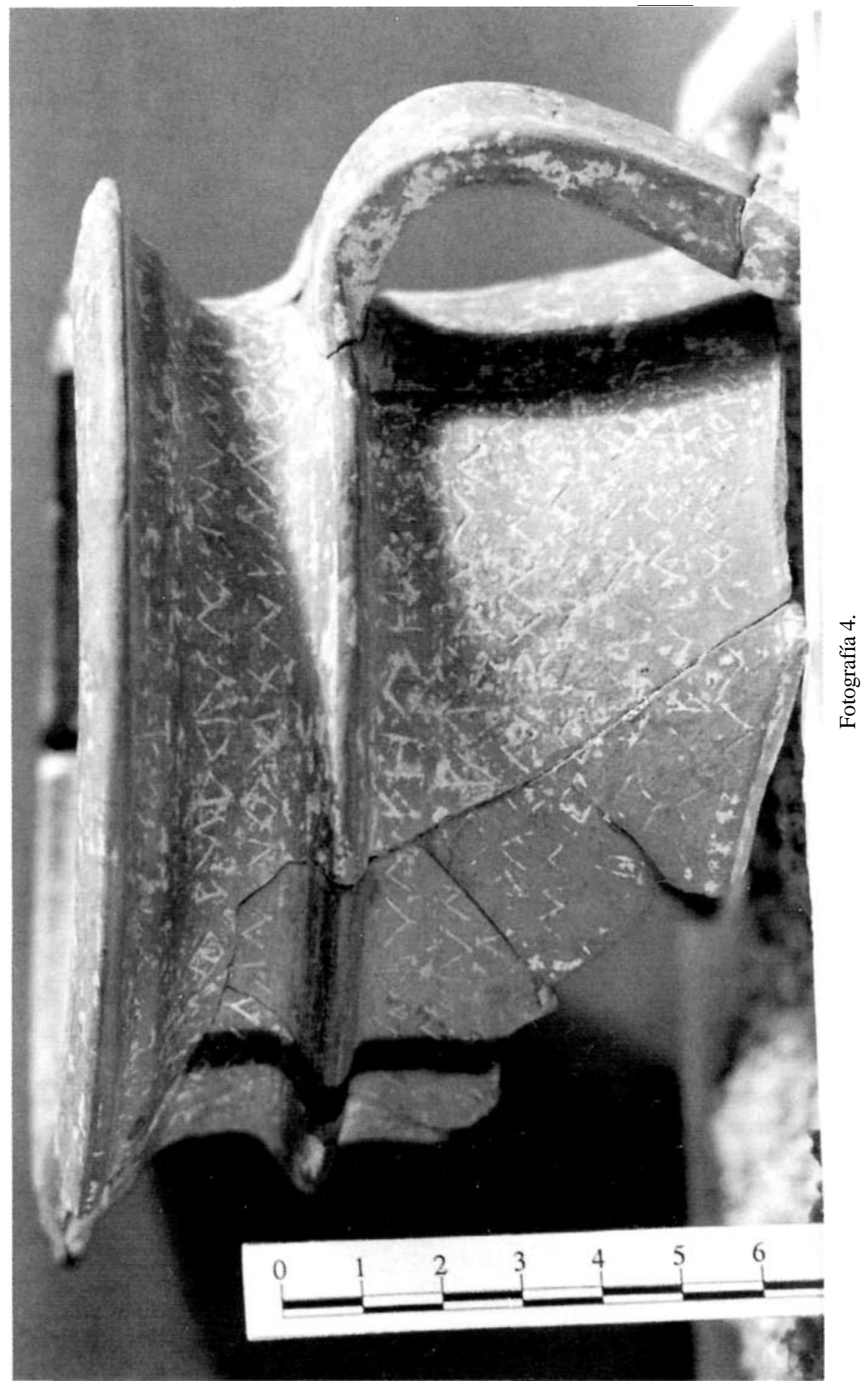


Inscripción ibérica procedente de La Joncosa (Jorba, Barcerlona)

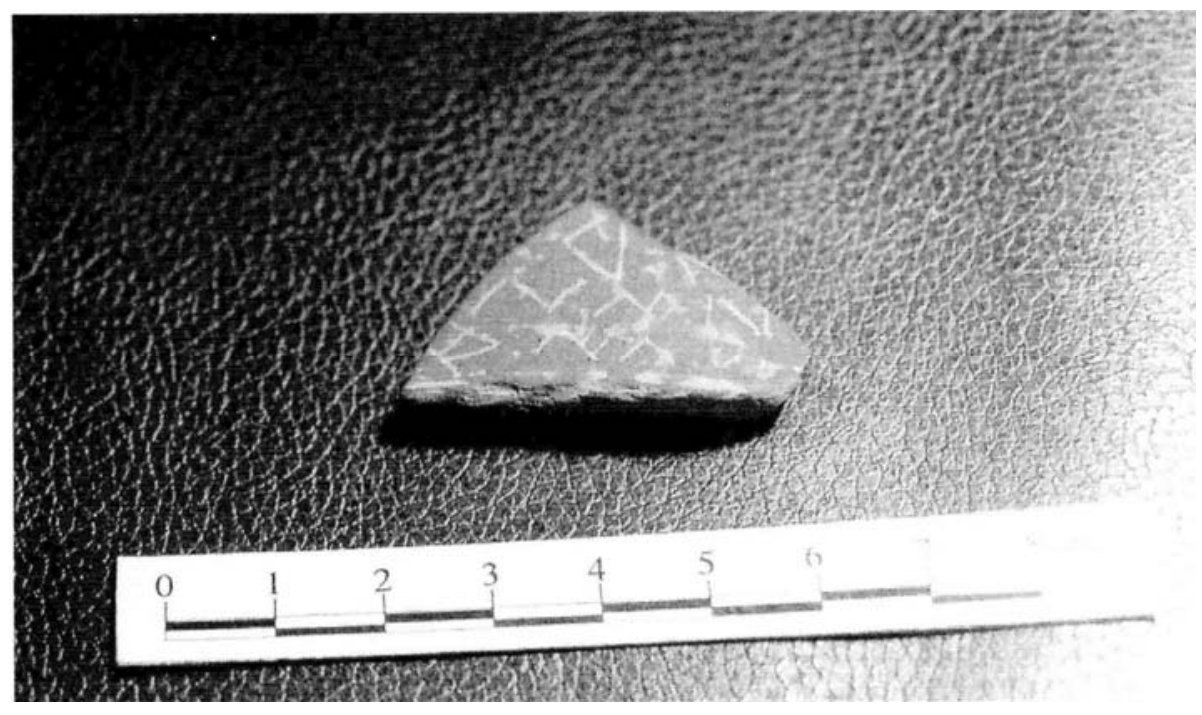

Fotografía 5. 